\title{
Contribución de las empresas cooperativas a la construcción de un mundo mejor
}

\author{
Siegbert Rippe
}

Sumario: I. Introducción. II. El pasado. III. El presente: 3.1. Generalidades. 3.2. Las cooperativas y (o vs) el Estado. 3.3. La Ley General de Cooperativas. 3.4. El Cooperativismo y la educación. 3.5. El cooperativismo, ¿lejos de la ciencia y la tecnología? 3.6. Los valores cooperativos en el mundo de hoy. IV. El futuro. Bibliografía.

Resumen: El presente trabajo pretende, en el Año Internacional de las Cooperativas, así declarado por la ONU, exponer sintéticamente, con base académica y estilo cuasi periodístico, sobre la contribución del cooperativismo y de las empresas cooperativas de Uruguay a la construcción de un mundo mejor, mediante el tratamiento igualmente sintético de su pasado y de su presente y el abordaje de aspectos tales como la teoría y práctica de los valores cooperativos vinculándolos, en tal marco de referencia, con otros tales como el cooperativismo y el Estado, la nueva, reciente legislación orgánica de las cooperativas, el cooperativismo y, por un lado, la educación y, por el otro, la ciencia y la tecnología, para luego ingresar en la abreviada y conclusiva consideración sobre aquellos valores en el presente y exponer en definitiva ciertas, básicas ideas sobre el próximo futuro, todo ello, en tanto relacionados con la construcción de un mundo mejor mediante el quehacer de las cooperativas y en la convicción o, por lo pronto, con la expectativa de su contribución a tal construcción.

Palabras clave: Cooperativas/ mundo mejor; cooperativas y cooperativismo en Uruguay; pasado, presente y futuro de las cooperativas; Cooperativismo y cooperativas: desafíos y oportunidades; empresas cooperativas: perspectivas

Abstract: With this work, in the International Cooperative year, declared by UN, we will synthetic expose, with academic knowledge and sort of journalistic style, about the Cooperatives and Cooperative companies in Uruguay contribution in the construction of a better world. We will expose, also synthetic, about its past, present and the treatment from aspects such as theory and practice of the cooperatives values and relating them with different actors, such the Estate, the new organic cooperative's law, education, science and technology, to finally get into a briefly and conclusive consideration about 
the actual values and expose basic ideas about the future of cooperatives, all related with the construction of a better world through cooperatives and the conviction and therefore, the expectations of the cooperative's contribution for this construction.

Key words: Cooperatives- Better world; cooperatives in Uruguay; past, present and future of cooperatives; cooperatives: challenges and opportunities; cooperative companies: perspectives. 


\section{Introducción}

El 2012 es el Año Internacional de las Cooperativas y en el marco de una temática cuyo título y lema son «Las empresas cooperativas construyen un mundo mejor», se analizará brevemente la situación de las mismas en Uruguay, para así concluir luego cómo y cuánto las cooperativas se están desarrollando y desenvolviendo en pro de un mundo mejor. Por esta razón se hará primero, una breve reseña del pasado, luego, un paneo del presente y, por último, una aproximación a las posibles vías del futuro de las cooperativas para que en la teoría y en la práctica logren llevar adelante la propuesta de «construir un mundo mejor».

Se considera que la mejor forma de comenzar, es comprender el mundo de las cooperativas. Cooperar es un verbo que expresa y define en el caso un movimiento que implica solidaridad, trabajo en equipo y relativo desinterés económico en lo estrictamente individual. Las cooperativas son una parte instrumental de este movimiento y como tales deben seguir sus valores y principios y promoverlos dentro de la sociedad en la que se insertan.

Los valores cooperativos según la Alianza Cooperativa Internacional $(\mathrm{ACl})$, son los siguientes: la ayuda mutua, responsabilidad, democracia, igualdad, equidad y solidaridad ${ }^{1}$.

Pero en el siglo XXI, con los nuevos adelantos tecnológicos, la competencia global y un mundo que se mueve de manera vertiginosa, se impone una pregunta ¿cómo pueden seguir siendo reales estos valores, principalmente el de solidaridad?

A tales efectos se hará seguidamente una suerte de sinopsis que incluirá algunos pantallazos sobre: el pasado y presente del cooperativismo y de las cooperativas uruguayas, las cooperativas y el Estado, la ley general de cooperativas, el cooperativismo y la educación, el cooperativismo y la ciencia y la tecnología para considerar después los valores cooperativos en el mundo de hoy e introducirse finalmente en el futuro del movimiento y de las cooperativas.

La metodología expositiva empleada en la oportunidad, atento a las características de la temática a tratar, procura combinar una necesaria base académica con una conveniente presentación de corte cuasi periodístico, en la convicción que tal combinación permitirá un mejor acercamiento a tal temática.

\footnotetext{
1 http://www.caycpa.com.uy/cooperativa.htm
} 


\section{El pasado}

En 1889 se reconoce la primera cooperativa de consumo, fundada en la Ciudad Vieja de Montevideo, principal puerto del país, sin embargo no es hasta 1920 que surge realmente el cooperativismo en lo formal y en lo material, en el que las cooperativas de consumo predominaban pero nacían también nuevas cooperativas como las ferroviarias y UTE (Usinas Eléctricas del Estado. CUTE). No obstante, es recién entre los años 1940-1950 que surge y se implementa la legislación propia y específica del movimiento cooperativo, a través de la determinación, definición y regulación de específicos tipos cooperativos (agropecuarias, de producción, de consumo), a las que luego se agregan otros (de ahorro y crédito, de vivienda, sociales, etc.).

En la publicación El cooperativismo en Uruguay, se describe breve y consistentemente el pasado cooperativo nacional: «...la historia del cooperativismo uruguayo tiene una trayectoria diferenciada según los grupos de interés que involucra.

Sin embargo postulamos que es posible reconocer algunos elementos coincidentes en esa trayectoria:

- La vinculación con organizaciones o gremiales. El papel determinante del Estado, tanto en su fomento como en la obstaculización de su desarrollo.

- El aprovechamiento de diversos espacios de actividad que han dejado las diversas modificaciones en el rol del Estado.

- La fuerte presencia en el interior del país.

- Su marcada autonomía y pluralidad. ${ }^{2} \gg$

En junio de 1988 se firma el documento de creación de la Confederación Uruguaya de Entidades Cooperativas, CUDECOOP, aunque en su momento bajo la forma de una asociación civil, que tiene como fin «la organización de representación y defensa gremial del movimiento cooperativo uruguayo. ${ }^{3}$, con manifiesta intención de fortalecer el cooperativismo a través de la unificación asociativa y voluntaria de todos lo tipos y formas cooperativas, cualquiera fuere su objeto o actividad económica o gremial.

2 Bertullo, Jorge; Isola, Gabriel; Castro, Diego; Silveira, Milton. El Cooperativismo en Uruguay, Universidad de la República, 2003, p. 7.

$3 \mathrm{http}: / / \mathrm{www}$.cudecoop.coop/index2.php? controlador=noticia\&accion=ver\& noticia $=129$ 


\section{El presente}

\subsection{Generalidades}

Uruguay tiene alrededor de un siglo de vida de cooperativismo, y como tal hay ciertas características que son importantes remarcar.

a) El Movimiento Cooperativo, tiene desde sus orígenes hasta la actualidad una vinculación directa con los movimientos sociales. Entendiendo por éstos los gremios, sindicatos y diversas organizaciones laborales, entre otros.

b) El cooperativismo en Uruguay ha tenido un nacimiento y un desarrollo muy marcado en el interior del país, teniendo una participación de casi $70 \%$ sobre el total de cooperativas. Este es un dato muy importante si tenemos en cuenta que la mitad de la población del país, vive en Montevideo, la capital del país.

c) Las cooperativas en Uruguay abarcan varias ramas de productos y servicios, incluyendo alimentos, transporte, servicios médicos, ahorro y crédito, vivienda, agrarias y de consumo, entre otras.

d) En Uruguay existen casi 1.260 entidades cooperativas, con algo más de 800.000 socios $^{4}$. Esto significa que un $25 \%$ (estimativo ya que muchos socios pertenecen a más de una cooperativa a la vez) de la población tiene una relación directa con el cooperativismo.

e) Las cooperativas se distribuyen a lo largo y ancho del Uruguay teniendo una distribución variable según el objeto principal de su fundación. Por ejemplo, las cooperativas agrarias y de producción son mayoritarias en el interior del país, mientras que en la capital predominan las de ahorro y crédito y, las de vivienda. El número de cooperativas médicas y de consumo es bastante similar en Montevideo y el resto del la República Oriental ${ }^{5}$.

Esta presencia del cooperativismo en el interior ha significado un muy importante aporte al desarrollo económico y social del país ya que la generación de puestos de trabajo, movimientos económicos y agrupaciones ha significado el crecimiento de varios sectores, descentralizando las agrupaciones de la capital.

La influencia de las cooperativas y su relación con la sociedad, el Estado, la educación y las investigaciones y tecnologías son los puntos si-

5 Primer Relevamiento de Entidades Cooperativas 1989, MTSS. ICS UDELAR. 
guientes que se resumirán para comprender más, o por lo menos mejor, la situación de las cooperativas y sus relaciones con los diferentes actores sociales.

\subsection{Las cooperativas y (o vs) el Estado}

Las cooperativas y el Estado son natural y razonablemente independientes entre sí, lo que ha llevado desde su creación hasta la actualidad, que tengan o mantengan una relación oscilante o con altibajos, pasando por momentos, a veces, de choque, otros, de convivencia armónica y coherencia finalística, épocas de alejamientos y acercamientos condicionados por la actitud de la administración y el gobierno de turno, la situación socio-económica del país y la posición estructural o coyuntural asumida por las cooperativas y los cooperativistas mismos.

En la publicación, ya antes mencionada, El Cooperativismo en Uruguay, se describe la relación de las cooperativas con el Estado en su momento como «... aún en construcción. En efecto, los impulsos de alguna manera convulsivos de la legislación nacional y la propia acción de los actores cooperativos no han logrado consolidar espacios de articulación satisfactorios, al menos a juicio de las organizaciones representantes del universo cooperativo del Uruguay» ${ }^{6}$.

En marzo de 1991 se creó por Decreto del Poder Ejecutivo la Comisión Honoraria del Cooperativismo $(\mathrm{CHC})$ que funcionó en la Oficina de Planeamiento y Presupuesto. Integrada por cinco miembros, tres de ellos designados por el Presidente de la República, y otros dos designados también por el Presidente pero seleccionados de una nómina de seis a sugerencia de la Confederación de Cooperativas (CUDECOOP). Los cometidos de la CHC fueron: velar por el cumplimiento de los principios cooperativos; promover y fomentar el desarrollo del movimiento cooperativo; asesorar y colaborar en la implementación de una política nacional; brindar asistencia técnica a las cooperativas, organizar un servicio estadístico y de difusión cooperativos, analizar y proponer reformas en materia del marco normativo?.

En una publicación de todavía reciente data se hace referencia a la realidad de las cooperativas uruguayas y el desarrollo promovido por el Estado expresando que: "... de todos los programas relevados pocos constituyen verdaderas políticas de fomento del cooperativismo... el

\footnotetext{
6 Id. cit. 2, p. 31.

7 www.chc.gub.uy
} 
objetivo último era facilitar el desarrollo, expansión y consolidación de las cooperativas en el tejido económico productivo... el cooperativismo se presenta muchas veces como una solución pero no para los cooperativistas sino que para el Estado, quien, a través de la gestión de las cooperativas, busca soluciones a sus propios problemas». Además, se remarca «la insuficiencia de las respuestas del Estado y, en todo caso, la parcialidad de sus respuestas» ${ }^{8}$, asumiéndose una posición entendible aunque no necesariamente compartible en su totalidad, en la oportunidad.

\subsection{La Ley General de Cooperativas ${ }^{9}$}

En el año 2008 se sancionó la nueva Ley General de Cooperativas, que modificó sustancialmente el panorama legislativo y posibilita una mejora en las perspectivas de cambio del cooperativismo. En esta ley se procura mantener y garantizar el conveniente equilibrio entre la autonomía de las cooperativas y la propia de los actores comprometidos con su promoción y control, así como regular el alcance de la participación e intervención normativa y operativa sobre las mismas por parte del Estado. La ley busca evitar la fragmentación del sector cooperativo e impulsar el reconocimiento de su unidad básica, generar certezas y seguridad jurídica para quienes contratan con las cooperativas, alcanzar la congruencia entre las metas de las cooperativas orientadas hacia el desarrollo y su autosuficiencia y sustentabilidad, facilitar la cooperación e integración cooperativas; facilitar procesos de integración regional, e; incrementar y mejorar los instrumentos de capitalización, entre otros.

Sin embargo, esta nueva Ley sigue sin promover orgánica y consistentemente políticas estatales específicas de promoción de las cooperativas de forma continua y transversal, siendo todavía movimientos e instrumentos puntuales los que incentivan el desarrollo cooperativista.

Además, se ha advertido que ciertos temas no han sido especificados en esta nueva Ley ${ }^{10}$ :

8 MARTI, Juan Pablo. Evaluación de la legislación y los programas de fomento del cooperativismo en Uruguay. Esfuerzos espasmódicos y reglamentarios. Montevideo, noviembre de 2010, p. 16.

9 RIPPE, Siegbert. «Innovaciones introducidas en la legislación cooperativa de la República Oriental del Uruguay por la nueva Ley General de Cooperativas». Boletín de Derecho Cooperativo, Universidad de Deusto, 2009.

10 Id. cit. 6, p. 22. 
— la posibilidad de la creación de cooperativas de incidencia social que sean agrupadas por personas marginadas de la sociedad;

- la creación de cooperativas de técnicos, empresarios y/o profesionales que presten sus servicios a la comunidad (a excepción de las cooperativas ya existentes en las áreas de salud y educación).

Por otra parte, el Estado ha fomentado el desarrollo de nuevas cooperativas a través de los Ministerios de Vivienda, Ordenamiento Territorial y Medio Ambiente, (cooperativas de vivienda) y de Desarrollo Social (cooperativas sociales) ${ }^{11}$ y realizó un plan de sensibilización para que los cooperativistas lleven adelante nuevos y más emprendimientos productivos en todo el territorio nacional con el apoyo de la Federación de Cooperativas de Producción del Uruguay.

\subsection{El Cooperativismo y la educación}

Desde hace mucho tiempo se pretende que el cooperativismo sea parte de la educación nacional desde el nivel primario hasta el universitario.

En 1926 el Maestro Julio Marrote expuso ante autoridades de la Enseñanza Primaria la importancia de promover el cooperativismo a los niños, como parte del programa educativo. Recién en 1958 se decidió crear una Comisión Especial para orientar y supervisar el cooperativismo escolar. Esto logró que varias escuelas lleven adelante experiencias donde los estudiantes ejercían el rol de cooperativistas. En 1969 se creó la Oficina Central del Cooperativismo Escolar para organizar y colaborar con emprendimientos escolares cooperativistas. Pese a que esta comisión lleva más de medio centenar de años funcionando (con un cese de actividades temporario durante la dictadura -1973-1984-), son pocos los éxitos cooperativistas escolares, siendo aislados tanto por sectores geográficos como por continuidad durante los años lectivos ${ }^{12}$. Más allá de esto, en las escuelas se hace referencia al movimiento cooperativo como parte del programa escolar.

El vínculo entre las cooperativas y la educación media se siente también inestable y tiene esfuerzos aislados para una implementación

11 www.larepublica.com.uy/economia/364223-impulso-al-desarrollo-cooperativo

12 CUDECOOP. La reforma de los estatutos de las cooperativas en función de los cambios e innovaciones derivados de la Ley General n. ${ }^{\circ} 18.407$, mayo de 2011, pp. 3839. 
exitosa. El mayor esfuerzo ha sido el del Consejo de Educación Técnico Profesional (organización que capacita en varias áreas a los jóvenes para darles herramientas prácticas con salida laboral), que tiene desde 1967 una Comisión que promueve el cooperativismo juvenil, formando así a estudiantes y docentes en materia cooperativa, resaltando la importancia de las cooperativas de trabajo.

En la educación universitaria el desarrollo de la enseñanza del cooperativismo ha sido aparentemente más notoria y trascendente. En efecto. La Universidad logró participar activamente en el Primer Censo Nacional de Entidades Cooperativas, en una investigación sobre la incidencia del Cooperativismo en la economía nacional y en la creación de la Unidad de Estudios Cooperativos en el contexto administrativo y funcional de aquella, entre otros eventos institucionales. Mientras que, además, busca participar e incrementar las relaciones con otros países siendo integrante de la Red Universitaria de las Américas con Estudios en Cooperativas y Asociativismo (UNIRCOOP), del Comité Académico sobre Procesos Cooperativos y Entidades Económicas Asociativas (PROCOAS) de la Asociación de Universidades del Grupo Montevideo.

En el ensayo de esta autoría, "Cooperativismo y Universidad», se define la relación de las cooperativas con la Universidad sosteniendo que: «Desde el punto de vista del movimiento cooperativo es notorio el interés del mismo por los avances en materia de investigaciones y cursos de y para el Cooperativismo, así como la disponibilidad de diferentes agentes de las cooperativas por colaborar y participar en cursos, seminarios, investigaciones y formación de los universitarios. Sin embargo, 20 años después del Cursillo realizado en 1987 las cooperativas siguen viendo algunos problemas y falencias con respecto a la Universidad. Estas falencias mencionadas son: la escasa formación que aún existe hoy en casi todas las carreras vinculadas a las posibilidades del desarrollo de las cooperativas; mayor número de investigaciones, consultorías y análisis concretos de la realidad actual en lo que se refiere a las cooperativas y su rol en la sociedad y en las políticas estatales a nivel nacional e internacional, y el débil vínculo entre la Universidad y las cooperativas ${ }^{13}$. La situación no ha cambiado de manera relevante en estos últimos años, más allá de algunos avances puntuales en algunas áreas universitarias, particularmente de la universidad pública.

De todas formas, se considera que lo más importante a destacar son ciertos cambios de la educación pública en la sociedad uruguaya y,

13 RIPPE, Siegbert, «Cooperativismo y Universidad», Boletín de la Asociación Internacional de Derecho Cooperativo, Universidad de Deusto, 2008. 
por ende, el rol más activo y participativo que se observa en la educación privada.

Al respecto y en una publicación titulada "Características sociales, políticas y culturales de la sociedad uruguaya», se define la situación actual de la educación en el país en estos términos: "La educación pública ha perdido gradualmente su función integradora a medida que se extiende la franja social que acude a la educación privada. Si antes de la dictadura la opción por la educación privada era elegida solo por una minoría, muchas veces por razones religiosas o lingüísticas, durante la dictadura se volvió una de las pocas vías para lograr una educación de calidad en un ambiente de respeto y amabilidad, lejos del autoritarismo de los «porteros-policías» que imperaban en los liceos públicos. La extensión de la pobreza y la exclusión, fue concomitante con la paulatina deserción de la clase media de las instituciones públicas de Montevideo (no así en el interior) ${ }^{14}{ }^{14}$.

Este nuevo panorama de la educación debería ser tenido en cuenta tanto por el Estado como por las cooperativas para lograr que los estudiantes en general, tengan acceso al conocimiento del mundo cooperativo, a la formación de cooperativas educativas y a entender esta realidad como una oportunidad de integración, donde los segmentos sociales y educacionales se unan a través de cooperativas que los ayuden a crecer y desarrollarse.

\subsection{El cooperativismo, ¿lejos de la ciencia y la tecnología?}

En Uruguay casi toda la investigación científica, por lo pronto, la más significativa y así reconocida, se realiza en la Universidad de la República. La investigación se financia a través de fondos concursales centrales de la Universidad, horas de investigación por la vía del Servicio (dependencia universitaria) a la cual pertenece el investigador, y gracias a posibles convenios entre el investigador y sectores públicos y privados $^{15}$. La realidad es que más allá de los esfuerzos, las investigaciones relacionadas al mundo cooperativo son escasas y esporádicas, siendo únicamente las facultades de agronomía y veterinaria quienes se destacan por las investigaciones vinculadas al sector cooperativo.

Además, en un mundo donde las TICX (Tecnologías de la Información, Comunicación y Conectividad) tienen un rol protagónico con

14 Isabel CLemente en http://www.quehacereducativo.edu.uy/docs/fdacc61e_22\%20 formaci\%C3\%B3n-a.pdf

15 ld. cit. 5, p. 39. 
avances continuos, las cooperativas deberían ser parte de esta modalidad, tanto como la formación de nuevas cooperativas centradas en la tecnología como en contar con todas las herramientas necesarias para que las TICX y su uso responsable sean parte tanto de la infraestructura, el material y la educación en todas sus variables.

Se puede entonces extraer que salvo por investigaciones particulares y de rubros específicos las cooperativas y el desarrollo científico, las cooperativas y el mundo de las TICX están aún distanciados, tanto en el desarrollo cooperativo en éstas áreas, como muchas veces, en el accesos a recursos tecnológicos que las ayuden a competir a nivel nacional e internacional.

\subsection{Los valores cooperativos en el mundo de hoy}

Al inicio de este ensayo se mencionaron los valores cooperativos, ahora es tiempo de analizar cómo se conllevan éstos con la realidad del siglo XXI y sus continuos avances:

a) Ayuda mutua. El concepto de ayuda mutua significa intercambiar de forma natural por la propia necesidad del ser humano de socialización, experiencias y conocimiento donde se da y se recibe de igual forma ${ }^{16}$. Los uruguayos siguen tendiendo a pertenecer a grupos sociales y sobre todo los jóvenes a redes sociales que les dan el sentido de pertenencia. La ayuda hacia el otro, existe pero en su mayoría se da en el campo de la afinidad ideológica, la comunidad religiosa y del grupo social de pertenencia, potenciándose la fragmentación en todos sus aspectos. Además, la violencia, falta de respeto y la delincuencia inciden en la limitación de la ayuda mutua, ya que: uno, el ser humano por sí mismo busca auto protegerse para subsistir y; dos, al mismo tiempo, participa en muchas ocasiones en organizaciones para proteger al prójimo. ${ }^{17}$ Fundamentalmente colabora en forma sistemática con aquellas asociaciones civiles que son reconocidas y aceptada por sus objetivos, que rinden cuenta de los apoyos y tienden el respaldo de su responsabilidad social.

b) Responsabilidad. Este término hace referencia a la responsabilidad social que tienen las cooperativas con la comunidad. Sin embargo, este atributo característico en las cooperati-

16 http://www.feacab.org/docs/familia.pdf

17 ld. cit. 14. 
vas es ahora también parte de los planes accionarios de empresas públicas y privadas. Siendo este un atributo tan importante que, según un informe de Presidencia de la República, el $83 \%$ de la población uruguaya opta por consumir en empresas que fomenten la responsabilidad social ${ }^{18}$, como por ejemplo: Mc Donald's, Movistar, Disco (supermercados), etc.

c) Democracia. El control democrático de las cooperativas es fundamental para su desarrollo y sobre todo para el cumplimiento de uno de los principios fundamentales del cooperativismo. Sin embargo, en un artículo titulado "Cooperativas, democracia, recursos humanos y género: ¿qué tienen que ver uno con el otro», se señala: "Las cooperativas son organizaciones democráticas controladas por sus miembros, quienes participan activamente en la definición de las políticas y en la toma de decisiones. Los hombres y mujeres elegidos para representar a su cooperativa responden ante los miembros. En las cooperativas de base los miembros tienen igual derecho de voto (un miembro, un voto), mientras que en las cooperativas de otros niveles también se organizan con procedimientos democráticos. En el pasado, y todavía ahora, "democracia» parece significar en la realidad: igualdad entre algunos y exclusión para el resto» ${ }^{19}$. Dura crítica a la práctica de la democracia en las cooperativas, que indica que los derechos de participación, voto y representación no cumplen con el verdadero significado de la palabra democracia, en tanto existen reales discriminaciones, incluso, hasta de género.

d) Igualdad. Frente a lo expuesto en la realidad de la democracia de las cooperativas se concluye que hay una discriminación tanto de género, como de clase social e ideologías políticas. Por ende, la igualdad dista mucho de su significado que según la Real Academia Española es «Principio que reconoce a todos los ciudadanos capacidad para los mismos derechos» ${ }^{20}$.

e) Equidad. La equidad es por otra parte la justa distribución de los excedentes entre los miembros de la cooperativa ${ }^{21}$.Principio que apunta a reafirmar que las cooperativas son por y para el bienestar de sus integrantes y que no tienen fines de lucro.

18 Lieve Daeren,en http://archivo.presidencia.gub.uy/_Web/noticias/2007/03/ 2007032108.htm

19 http://www.neticoop.org.uy/article385.html

20 www.rae.es

21 http://es.wikipedia.org/wiki/Movimiento_cooperativo 
f) Solidaridad. Esta gran palabra implica que las cooperativas como movimiento se mantienen unidas con el fin de ser parte de la sociedad en su conjunto, facilitando el acceso a recursos.

Los principios cooperativos son por su parte expresiones llenas de significado, en tanto reglas imperantes para la efectiva realización de esos valores. Sin embargo, la realidad de las cooperativas en muchos aspectos dista de la ejecución de los mismos. Estos principios, ajustados a la realidad, y su énfasis en respetarlos son la base para que las cooperativas en su quehacer, se constituyan en un movilizador del cooperativismo, que se refleje en un movimiento real que avanza a construir un mundo mejor.

Antes de abocarse a mirar hacia adelante es importante resaltar la situación social del Uruguay, situación que debemos enfrentar, conciliar y comprender para poder adaptar la sociedad al mundo cooperativo y viceversa.

En la publicación, antes mencionada, su autora expone: "La sociedad uruguaya se caracteriza hoy por la fragmentación. Contra la cualidad de integración y homogeneidad que prevaleció en la mayor parte del siglo XX» ${ }^{22}$, destacando los nuevos valores culturales, los nuevos grupos de integración, el cambio de roles de las familias como detonantes de una sociedad que está cada día más desconfiada y donde lo que busca es auto protegerse. Aquí, entonces las cooperativas pueden ver una oportunidad integradora. En este contexto: ¿qué papel integrador podrían jugar las cooperativas?

\section{El futuro}

Dos preguntas cabe inicialmente formular... y contestar

¿Cómo puede ser el futuro de las cooperativas? y, ¿pueden éstas cooperar efectivamente a construir un mundo mejor, por lo pronto, en el ámbito de Uruguay?

Con la nueva Ley del cooperativismo ${ }^{23}$ se crea el Instituto Nacional del Cooperativismo (INACOOP) que está regulado por el Derecho Privado -es una persona pública no estatal, esto es, una entidad paraestatal - y que tiene como objetivo «promover el desarrollo econó-

23 ld. cit. 9. 
mico, social y cultural del sector cooperativo y su inserción en el desarrollo del país» (Ley 18.407), lo que genera la expectativa de una nueva posibilidad para el crecimiento y la promoción del mundo cooperativo. INACOOP no tiene como objeto la regulación y control estatal de las cooperativas, sino participar proactivamente en el rol de éstas en lo económico y social y en el desarrollo de su potencial en el siglo XXI. Este instituto obtendrá fondos por un lado, con base en aportes de las propias cooperativas y, por el otro, del Estado a través de recursos previstos en el presupuesto nacional, lo cual le brindará herramientas económico-financieras que le permitirán actuar de forma continua en el cumplimiento de sus objetivos.

Las cooperativas uruguayas tienen mucho pasado, tienen también un presente que quiere construir y consolidar el sentido cooperativo, en un mundo globalizado. Se han desarrollado en todo el territorio nacional, tienen una nueva Ley con un nuevo Instituto, se están desarrollando en rubros que van desde el agrícola hasta el médico, pasando por cooperativas de ahorro y crédito, vivienda, consumo y de transporte entre muchas otras, logrando así que formen parte de ellas o que las utilicen un estimado del $25 \%$ de la población nacional.

Pero, todo esto no es suficiente. Es necesario y conveniente generar estímulos de diverso tipo para fomentar el desarrollo de las cooperativas, en todas las áreas y que todas éstas tengan una visión acorde con los principios del cooperativismo, que realmente los estimule. También que mantengan una relación con el Estado clara y continua donde los esfuerzos no sean esporádicos y oportunistas, sino parte de un trabajo diario donde no sólo se busque fomentar el crecimiento de las cooperativas y su inserción en los tiempos veloces del siglo XXI sino que además, integren el sentido real de cooperar entre ellas, con la sociedad y en pro de un país en avance.

Las cooperativas son locales, en un sentido mere geográfico, constitutivo y operacional pero deben internacionalizarse (sin perder su identidad), abrir sus puertas, competir de forma leal en el comercio y los servicios mundiales, actualizarse y renovarse, crecer y abrir las puertas a los jóvenes ofreciéndoles un modelo de empresa diferente donde se resaltan también los valores de integración y de solidaridad, donde aquellos puedan adquirir experiencia y seguir profesionalizándose, si las cooperativas abren sus puertas y entablan una relación entre los experimentados y los inexperientes ${ }^{24}$.

24 ld. cit. 11. 
El Estado, pese a la independencia usual del cooperativismo de éste, debe coordinar de forma cotidiana los diferentes planes, programas y acciones del movimiento socio-económico mismo, teniendo una mejor y mayor comunicación transversal entre las entidades estatales y paraestatales que de forma singular propician planes cooperativistas.

Además, es importante considerar la formación continua, incluyendo intercambios regionales e internacionales de los cooperativistas en todos los rubros y niveles, con el fin de aprender de las prácticas y las experiencias en un mundo que es dinámico y que exige que las cooperativas se aggiornen a él para crecer y no perecer.

Tal vez la mejor forma de terminar esta colaboración es contando una anécdota personal. Mientras la escribía, un domingo, una de mis nietas (Micaela de 9 años), se acercó a mí en una reunión de familia y me preguntó: ¿qué es el cooperativismo?, ¿cuáles son sus principios?. Sorprendido, le pregunté: ¿Por qué quieres saber eso?, y de forma muy natural me contestó «es el año internacional de las cooperativas y en la escuela nos mandaron deberes para que aprendamos más». La senté conmigo y le empecé a contar acerca del mundo cooperativo y su finalidad, y cuando ella empezaba a entusiasmarse con mi explicación me preguntó «¿yo uso las cooperativas?»,y nos pusimos a pensar y tomar nota de su vida y agenda diarias y la sorpresa fue grande y hasta incluso muy grata cuando llegamos a las conclusiones: usaba buses de cooperativas de transporte, se atendía en una cooperativa médica, consumía productos alimenticios de cooperativas de consumo e iba de compras casi diariamente a alguna de ellas y estudiaba con una compañera de clase que vivía en una cooperativa de vivienda, y jella no lo sabía! Como recién supo que sus padres, al comentarles sus hallazgos, le explicaron que tenían ahorros y tomaban préstamos en una cooperativa de ahorro y crédito.

Esta es una realidad, la realidad por lo menos, en el Uruguay, realidad que muestra que haciendo las cosas en conjunto con todos los actores de forma continua y sinérgica, comprendiendo que las cooperativas no son sólo empresas sino parte de un movimiento solidario, es que se puede llegar a potencializar las cooperativas, teniendo en cuenta que el momento es ahora, es hoy, no mañana y menos en años.

Si uno no se queda únicamente en el uso de las respectivas prestaciones de las cooperativas sino que además se aprende desde niños su perfil y papel en la sociedad y en la economía, sus principios y valores, si además el Estado mantiene un rol activo, continuo y coordinado y si las cooperativas les dan más y mejores oportunidades laborales a los jóvenes y también a otros sectores sociales usualmente vulnerables o marginados, se podrá decir que ellas ayudan a construir un mundo me- 
jor en el cumplimiento de los nobles valores y principios universales del cooperativismo sin distinción de género, y que hay un movimiento organizado que coopera en esa construcción.

\section{Bibliografía}

Bertullo, Jorge; Isola, Gabriel; CAstro, Diego; Silveira, Milton. el Cooperativismo en Uruguay, Universidad de la República, 2003.

CUDECOOP. La reforma de los estatutos de las cooperativas en función de los cambios e innovaciones derivados de la Ley General n. ${ }^{\circ}$ 18.407, mayo de 2011.

CUDECOOP, "Suplemento Cooperativismo», Los jóvenes: futuro de la empresa cooperativa, julio de 2011.

MARTI, Juan Pablo. Evaluación de la legislación y los programas de fomento del cooperativismo en Uruguay. Esfuerzos espasmódicos y reglamentarios. Montevideo, noviembre de 2010.

RIPPE, Siegbert. "Una vinculación necesaria y viable con el cooperativismo», en el Boletín de Estudios Cooperativos del SCEAM. UDELAR, año 1, número 1, junio de 1990.

- " "Universidad y cooperativismo», Boletín de la Asociación Internacional de Derecho Cooperativo, Universidad de Deusto, 2008.

- . «Innovaciones introducidas en la legislación cooperativa de la República Oriental del Uruguay por la nueva Ley General de las Cooperativas», Boletín de la Asociación Internacional de Derecho Cooperativo, Universidad de Deusto, 2009.

\section{Fuentes de información referencial}

http://www.youtube.com/watch?v=_ry_HETQVyY\&feature=player_embedded http://www.ilo.org/global/topics/employment-promotion/cooperatives/lang--es/ index.htm

www.larepublica.com.uy/economia

www.chc.gub.uy

www.cudecoop.coop

www.elpais.com.uy

www.wikipedia.org/wiki/Movimiento_cooperativo

www. neticoop.org.uy

www.rae.es

www.archivo.presidencia.gub.uy

www.feacab.org

http://www.quehacereducativo.edu.uy

www.mtss.gub.uy

http://www.caycpa.com.uy/cooperativa.htm 